\title{
Covering Orthogonal Polygons with Sliding $k$-transmitters
}

\author{
Salma Sadat Mahdavi* \\ Saeed Seddighin ${ }^{\dagger}$ \\ Mohammad Ghodsi ${ }^{\ddagger}$
}

\begin{abstract}
In this paper we consider a new variant of covering in an orthogonal art gallery problem where each guard is a sliding $k$-transmitter. Such a guard can travel back and forth along an orthogonal line segment, say $s$, inside the polygon. A point $p$ is covered by this guard if there exists a point $q \in s$ such that $\overline{p q}$ is a line segment normal to $s$ and has at most $k$ intersections with the polygon's boundary walls. The objective is to minimize the sum of the lengths of the sliding $k$-transmitters to cover the entire polygon. In other words, the goal is to find the minimum total length of trajectories on which the guards travel to cover the entire polygon. We prove that this problem is NP-complete and present a 2-approximation algorithm for it.
\end{abstract}

\section{Introduction}

We study a new version of the art galley problem to cover a simple orthogonal polygon where a new model of covering or visibility, using sliding cameras. Sliding camera guards were introduced by Katz and Morgenstern [8] for guarding orthogonal polygons. A sliding camera can travel back and forth along an axis-aligned segment $s$ inside an orthogonal polygon $P$. A point $p$ can be viewed by this camera if there exists a point $q \in s$ such that $\overline{p q}$ is a line segment normal to $s$ and is completely inside $P$. Another variation of coverage that we use for our guards in this paper is "Modem Illumination" where each guard is modeled as an omnidirectional wireless modem with an infinite broadcast range which can penetrate through $k$ (for a fixed integer $k>0$ ) walls to reach a client. These modems are also called $k$-transmitters and were introduced by Fabila-Monroy et al. [6] and Aichholzer et al. [1].

The sliding cameras which we used, can see through at most $k$ walls in the directions perpendicular to their line segment track. As we do here, the walls are most often represented by line segments with no diameters.

\footnotetext{
*Department of Computer Engineering, Sharif University of Technology, Tehran, Iran, smahdavi@ce.sharif.edu

$\dagger$ Department of Computer Science, University of Maryland, Maryland, USA, saeedreza.seddighin@gmail.com

${ }^{\ddagger}$ Department of Computer Engineering, Sharif University of Technology and Institute for Research in Fundamental Sciences (IPM) School of Computer Science, ghodsi@sharif.ir

$\S$ This author's work was partially supported by IPM under Grant No. CS-1392-2-01
}

The objective is to find the minimum-length sliding cameras that cover the entire polygon. This is our problem and we denote it as MLSC $k$. In this paper, we prove that MLSC $k$ is NP-complete and we present a 2approximation algorithm for it.

\section{Previous Work}

The art gallery problem is a classic area of study in computational geometry. Over the years, many variants of this problem have been studied [11,13], [7, 12]. Most of these variations are proved to be NP-hard [9], including the cases where the target region is a simple orthogonal polygon and the goal is to find the minimum number of vertex guards to guard the entire polygon $[11,12]$. Some variations with the limited model of visibility have polynomial time algorithms [10,14].

In [8], the authors considered the problem of guarding a simple orthogonal polygon with the minimum cardinality sliding cameras (MCSC). They showed that when the cameras are constrained to travel only vertically inside the polygon, MCSC can be solved in polynomial time. They also presented a 2-approximation algorithm for this problem when the trajectories which the cameras travel can be vertical or horizontal and the target region is an $x$-monotone orthogonal polygon. They left the computation of the complexity of MCSC as an open problem.

In 2013, Durocher and Mehrabi [5] studied the MCSC problem and the minimum-length sliding cameras (MLSC) problem with the goal to minimize the total length of trajectories along which the cameras travel. They proved that MCSC is NP-hard if the orthogonal polygon can have holes. They also proved that MLSC is solvable in polynomial time even for orthogonal polygons with holes. Recently, Durocher et al. [4] gave a (7/2)-approximation algorithm for MCSC.

In 2013, Ballinger et al. [2] considered the guards as $k$-transmitters. They extended bounds for the number of $k$-transmitters that are necessary and sufficient to cover a given group of line segments, polygons and polygonal chains.

\section{Notations}

Let $P$ be an orthogonal polygon. We refer to the area of $P$ with $\mathcal{A}(P)$ and its edges with $\mathcal{E}(P)$. We extend the 
endpoints of each edge $e \in \mathcal{E}(P)$ to obtain a line that contains $e$. Let $L$ be the set of these lines. Obviously, $L$ partitions $\mathcal{A}(P)$ into orthogonal rectangles denoted by $\mathcal{P}(P)$. For each sliding camera $c$, we denote $\mathcal{V}(c)$ as the set of points in $\mathcal{A}(P)$ which are guarded by $c$. Similarly, $\mathcal{V}^{k}(c)$ stands for the same set when we consider the problem using the $k$-transmitter model. We call a set of cameras $C$ a candidate set, if all points in each part $p \in \mathcal{P}(P)$ are covered with the same subset of $C$. We will prove that in MLSC $k$ there always exists an optimal solution which uses a candidate set.

\section{The Hardness of MLSC $k$ Problem}

In this section we prove that MLSC $k$ is NP-complete. We present a $\operatorname{poly}(n)$ reduction from the problem of tiling an orthogonal polygon by $1 \times 3$ rectangles to MLSC $k$. In the problem of tiling an orthogonal polygon with rectangles, it is assumed that the orthogonal polygon $R$ is drawn on a grid $G$. The goal is to place nonoverlapping $1 \times 3$ rectangles to cover all of $R$. Beauquier et al. showed that this problem is NP-complete [3].

Our proof has two phases. First, we construct a new orthogonal polygon $P$ from $R$. Next, we prove that for each answer to MLSC $k$ on $P$, there is a corresponding answer to the tiling problem on $R$. So, MLSCk is NPcomplete.

\subsection{The Reduction}

In this subsection we construct $P$ from $R$. The input for this construction is $R$ and a grid $G$. We denote the vertices of $R$ by $V_{R}$. Let $n$ be the number of grid vertices which are inside and on the boundary of $R$. Let $E_{G}$ be the set of edges of $G$ that are inside (not on the boundary) $R$, and $E E_{G}$ be the duplicated edges of $E_{G}$ (for each $e \in E_{G}$, there are $e, e^{\prime} \in E E_{G}$ ). We also denote the vertices of $G$ which are on the boundary of $R$ by $V B_{R}=\left\{v_{1}, \ldots, v_{n^{\prime}}\right\}$ (in clockwise order). At the end, we report the set $V_{P}$ which contains the set of all vertices and edges of $P$ in clockwise order.

We start traversing $V B_{R}$ from $v_{1}$, until we reach the first vertex $v_{i}$ such that $v_{i} \in V B_{R}$ and $v_{i} \notin V_{R}$. Then, we traverse $e_{i} \in E_{G}$ which is adjacent to $v_{i}$, and reach $v_{j}$. If $v_{j} \notin V B_{R}$, then we traverse the right most (in clockwise direction) adjacent edge of $v_{j}$, if it has not been traversed before. If all adjacent edges of $v_{j}$ have been traversed or if $v_{j} \in V B_{R}$, then we traverse the duplicated edge (traverse $e_{i}^{\prime}$ ).

We continue this process until we reach $v_{i}$ again. Then, we traverse the boundary of $R\left(V B_{R}\right)$ until we reach another un-traversed $v_{x}$ of $G$, which is not a vertex of $R$. We repeat the previous steps (traversing the adjacent edge of $v_{x}$, if it has not been traversed and performing the other steps) until we arrive back again to $v_{x}$. We traverse $R$ and $G$, until we reach $v_{1}$ again.

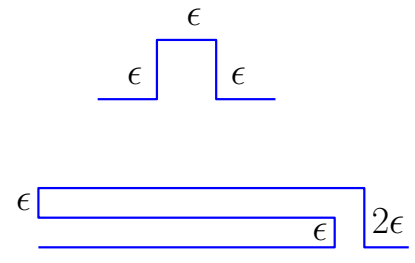

Figure 1: The added part

While traversing any vertex or edge, we add it to the set $V_{P}$. So, we construct a new polygon $P$ on $G$. We add some small parts to $P$ (see figure 1 ). These new parts are used to avoid having the sliding $k$-transmitters with the length greater than $1+\epsilon$ and to avoid having the transmitters which can cover two disconnected part of $P$. As we at most traverse all vertices and edges of $G$, the complexity of constructing $P$ is $O(n)$.

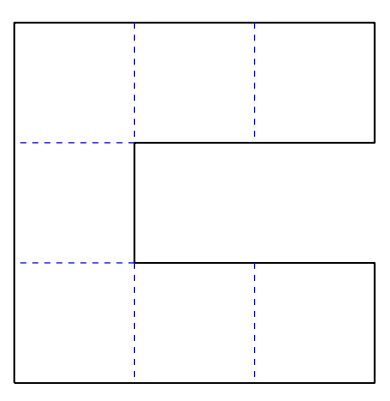

$R$

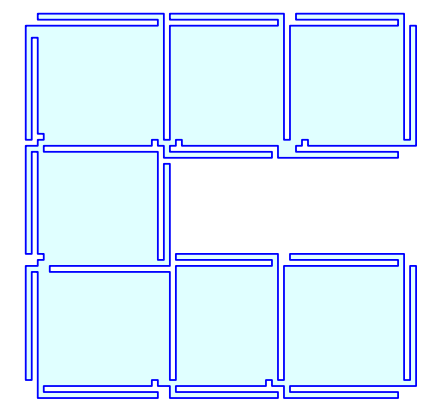

$P$
Figure 2: Construction of polygon $P$ from polygon $R$

\subsection{Correctness of the Reduction}

In this subsection we prove that for each answer of MLSC $k$ in $P$ there is an answer to the problem of tiling an orthogonal polygon $R$ with $1 \times 3$ rectangles and vice versa. So, MLSC $k$ is NP-complete. Let $g$, the number of the grid cells inside $R$, be a factor of 3 (otherwise $R$ can not be tiled by $1 \times 3$ rectangles). Also, let $k=2$, which means that the sliding transmitters can see through two walls.

First, assume that we solve MLSC $k$ on $P$ and its answer is denoted as $\left\{c_{1}, c_{2}, \ldots, c_{x}\right\}$. From the construction of $P$, the length of each transmitter can be $1 \pm \epsilon$ or $\epsilon$. Let $m$ the total length of the transmitters. If $m=g / 3+\epsilon$, then because of the construction of $P$ and the fact that each $c_{i}$ is a 2-transmitter, the answer to the tiling problem on $R$ is yes. Otherwise, the answer is no.

Second, assume that we solve tiling problem on $R$. Let $T=\left\{t_{1}, t_{2}, \ldots, t_{m}\right\}$ be the answer. We place the set of sliding $k$-transmitters $C_{1}=\left\{c_{1}, c_{2}, \ldots, c_{m}\right\}$ and $C_{2}$, which covers the entire $P$. From the construction of $P$, each rectangle $t_{i} \in T$ of $R$, is partitioned to three 
separated squares $s i_{1}, s i_{2}$, and $s i_{3}$ in $P$. We put a sliding $k$-transmitter $c_{i} \in C_{1}$ in the middle of $s i_{2}$ (see figure 3 ). Then for covering the added part (which are shown in figure 1), we put some transmitters with length $\epsilon$ or $2 \epsilon$ in $C_{2}$. As $c_{i}$ can see through at most two walls, so it covers only $s i_{1}, s i_{2}$, and $s i_{3}$. Since the rectangles are non-overlapping and they are tiling $R$, the set $C_{1}+C_{2}$ can cover the entire $P$ and $\left|C_{1}+C_{2}\right|=m+\epsilon$, the total length of transmitters, is minimal.

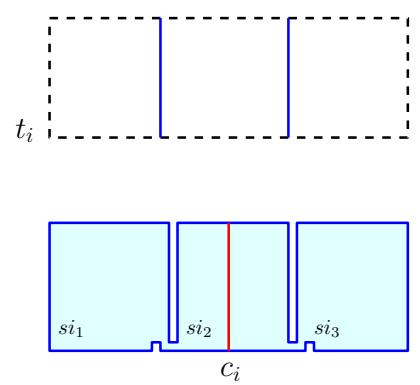

Figure 3: Placing the camera $c_{i}$ in the middle of rectangle $t_{i}$

So, we have the following theorem:

Theorem 1 The problem of covering a simple orthogonal polygon by the minimum-length sliding $k$ transmitters is NP-complete.

\section{Approximation Algorithm}

In this section we present a 2-approximation algorithm for MLSC $k$. The algorithm consists of two phases. In the first phase, we relax the problem to the case where each camera has a non-negative density. The goal is to put cameras in the polygon such that, the total density of cameras guarding each point is at least 1 and the total density of all cameras is minimized. Next, we present a polynomial time algorithm for the relaxed MLSC $k$ problem. In the second phase, we add some restrictions to the original problem regarding the answer of the relaxed MLSC $k$ problem on the given polygon. We prove that the objective function of the restricted problem is at most two times the objective function of the original problem. Moreover, we give a polynomial time solution for the restricted problem. At last, we report the solution of the restricted problem as a 2-approximation solution for the original problem.

\subsection{Relaxed MLSC $k$ Problem}

In this subsection we consider the relaxed version of MLSC $k$ and find an exact solution for this problem. The relaxed MLSC $k$ problem is defined as follows:

Definition 2 Given an orthogonal polygon $P$ and an integer number $k$. The relaxed $M L S C k$ problem is to put a set of sliding cameras $C=\left\{c_{1}, c_{2}, \ldots, c_{|C|}\right\}$ in $P$, each with density of $0 \leq d_{c_{i}} \leq 1$, in such a way that for every interior point $p \in \mathcal{A}(P)$, the following constraint is satisfied:

$$
\sum_{c_{i} \in C, p \in \mathcal{V}^{k}(c)} d_{c_{i}} \geq 1
$$

Among all solutions, the one that minimizes $\sum_{c_{i} \in C}\left|c_{i}\right| d_{c_{i}}$ is desired.

Let $\mathcal{R}(P)$ be $\sum_{c_{i} \in C}\left|c_{i}\right| d_{c_{i}}$ in an optimal solution of the relaxed MLSC $k$ problem on $P$. If we add the restriction that all $d_{c_{i}}$ s should be the in set $\{0,1\}$, the problem would be equivalent to the MLSC $k$ problem. Hence, $\mathcal{R}(P)$ is no more than $\mathcal{M}(P)$ (the optimal solution of the original problem) for all orthogonal polygons $P$.

Proposition 3 Any not-necessarily-optimal solution of the relaxed $M L S C k$ problem that the densities of all cameras are 1 is an acceptable but not-necessarily-optimal solution for MLSCk problem.

Proposition 4 For any given orthogonal polygon $P$ we have:

$$
\mathcal{R}(P) \leq \mathcal{M}(P) .
$$

Next, we show that the relaxed MLSC $k$ can be solved in polynomial time.

Lemma 5 There is a polynomial time algorithm that finds an exact solution for the relaxed MLSCk problem.

Proof. Let $\mathcal{C}(P)$ be a candidate set for the relaxed MLSC $k$ problem on the given orthogonal polygon $P$. There always exists an optimal solution for the problem using a subset of the cameras in $\mathcal{C}(P)$. The following linear program has $|\mathcal{C}(P)|$ variables $d_{c_{i}}$ for all $c_{i} \in \mathcal{C}(P)$.

$$
\begin{aligned}
& \min . \quad \sum_{c_{i} \in \mathcal{C}(P)}\left|c_{i}\right| d_{c_{i}} \\
& \text { s.t. } \quad \sum_{c_{i} \in \mathcal{C}(P), p \in \mathcal{V}^{k}\left(c_{i}\right)} d_{c_{i}} \geq 1 \quad \forall p \in \mathcal{A}(P) \\
& d_{c_{i}} \geq 0 \quad \forall c_{i} \in \mathcal{C}(P) \\
& d_{c_{i}} \leq 1 \quad \forall c_{i} \in \mathcal{C}(P)
\end{aligned}
$$

Constraints of type 2 state that each point in $\mathcal{A}(p)$ should be in the visibility area of cameras that the total sum of their densities is at least 1. Constraints of types 3 and 4 state that density of each camera is between 0 and 1 . The objective function is to minimize the total cost of all cameras where cost of each camera $c_{i}$ is defined as $\left|c_{i}\right| d_{c_{i}}$. Hence, the above LP finds an optimal solution for the relaxed MLSC $k$ problem. We remark that since $\mathcal{C}(P)$ is a candidate set of cameras for $P$, every point in each partition of $P$ is in the visibility 
area of the same set of cameras of $\mathcal{C}(P)$. Hence, we can rewrite the constraints of type 2 in the following way:

$$
\text { s.t. } \quad \sum_{c_{i} \in \mathcal{C}(P), p \in \mathcal{V}^{k}\left(c_{i}\right)} d_{c_{i}} \geq 1 \quad \forall p \in \hat{C}(P)
$$

The number of the variables and constraints of the LP is $\operatorname{poly}(n)$, therefore we can solve it in time $\operatorname{poly}(n)$.

\subsection{Restricted MLSC $k$ problem}

In the previous subsection we discussed the relaxed MLSCk problem and showed how can we solve it in polynomial time. Next, we define the restricted MLSC $k$ problem and show that this problem can be solved in polynomial time too.

Definition 6 Given an orthogonal polygon $P$ and an integer number $k$ and function $f: \mathcal{P}(P) \rightarrow\{H, V\}$, let $\mathcal{V}^{* k}$ be a function that for every horizontal camera $c$, $\mathcal{V}^{* k}(c)$ is the set of all partitions $p \in \mathcal{V}^{k}(c)$ such that $f(p)=H$. Similarly $\mathcal{V}^{* k}(c)$ for a vertical camera $c$ is the set of all partitions $p \in \mathcal{V}^{k}(c)$ such that $f(p)=V$. The restricted $M L S C k$ problem is to put a set of sliding cameras $C=\left\{c_{1}, c_{2}, \ldots, c_{|C|}\right\}$ in $P$, each with density $0 \leq d_{c_{i}} \leq 1$, in such a way that for every interior point $p \in \mathcal{A}(P)$, the following constraint is satisfied:

$$
\sum_{c_{i} \in C, p \in \mathcal{V}^{* k}(c)} d_{c_{i}} \geq 1
$$

Among all solutions, the one that minimizes $\sum_{c_{i} \in C}\left|c_{i}\right| d_{c_{i}}$ is desired.

Let $\mathcal{R}^{\prime}(P, f)$ be $\sum_{c_{i} \in C}\left|c_{i}\right| d_{c_{i}}$ in an optimal solution of the restricted MLSC $k$ problem on polygon $P$ and function $f$. We call a solution of the restricted MLSC $k$ problem Integral iff all of its guarding cameras have density 1. Next, we show that for every orthogonal polygon $P$ there exists a function $f: \mathcal{P}(P) \rightarrow\{\mathrm{H}, \mathrm{V}\}$ such that $\mathcal{R}^{\prime}(P, f) \leq 2 \mathcal{M}(P)$. Moreover, we show that such a function $f$ can be found in polynomial time.

Lemma 7 There exists a polynomial time algorithm that for every orthogonal polygon $P$ finds a function $f: \mathcal{P}(P) \rightarrow\{H, V\}$ such that $\mathcal{R}^{\prime}(P, f) \leq 2 \mathcal{M}(P)$.

Proof. Remark that, we can solve the relaxed MLSC $k$ problem for polygon $P$ in polynomial time. Let $C=$ $\left\{c_{1}, c_{2}, \ldots, c_{|C|}\right\}$ be the set of the cameras in an optimal solution of the relaxed MLSC $k$ problem and the density of camera $c_{i}$ be $d_{c_{i}}$. Moreover, our algorithm for the relaxed MLSC $k$ problem always selects a candidate set of cameras. We construct function $f: \mathcal{P}(P) \rightarrow\{\mathrm{H}, \mathrm{V}\}$ in the following way:

- For every partition $p \in \mathcal{P}(P)$, that the total densities of horizontal cameras guarding it is not less than $1 / 2$, we set $f(p)=\mathrm{H}$.
- We set $f(p)=\mathrm{V}$, for all other partitions $p \in \mathcal{P}(P)$.

Since the total sum of densities of all cameras guarding each point is at least 1 , for each partition $p \in \mathcal{P}(P)$ which $f(p)=\mathrm{V}$, the sum of densities of all vertical cameras guarding it is at least $1 / 2$. Now, we use all cameras $c_{i} \in C$ with densities $d_{c_{i}}^{\prime}=2 d_{c_{i}}$ as a solution for the restricted MLSC $k$ problem. Therefore, $\sum_{c_{i} \in C}\left|c_{i}\right| d_{c_{i}}^{\prime}=2 \mathcal{R}(P)$ and all the constrains of the restricted MLSC $k$ problem are satisfied. Hence, $\mathcal{R}^{\prime}(P, f) \leq 2 \mathcal{R}(P)$. Therefore, by Proposition (4) we have:

$$
\mathcal{R}^{\prime}(P, f) \leq 2 \mathcal{M}(P)
$$

To obtain a 2-approximation algorithm for the MLSC $k$ problem that runs in polynomial time, we show that every instance of the restricted MLSC $k$ problem has an integral solution which is optimal. Furthermore, we show that such an optimal integral solution can be found in polynomial time.

Lemma 8 There exists a polynomial time algorithm that finds an optimal integral solution for the restricted $M L S C k$ problem.

Proof. Since in the restricted MLSC $k$ problem each part of the polygon can be guarded with either vertical or horizontal cameras, we can divide the problem into two separate subproblems. In the first subproblem our aim is to put vertical cameras with minimum total length which guard all the parts of the polygon which can be guarded by vertical cameras. In the other subproblem we want to guard the remaining parts with horizontal cameras such that the total length of cameras is minimized. Since in both subproblems we have only horizontal or only vertical cameras, we can find the integral solutions in polynomial time. Combining the solutions of both subproblems gives us an optimal integral solution for the restricted MLSC $k$ problem.

Note that, from every integral solution of the restricted MLSC $k$ problem for orthogonal polygon $P$ and arbitrary function $f$, we can find a solution of the MLSC $k$ problem for polygon $P$ with the same set of cameras. Therefore, Lemmas (7) and (8) show that there exists a polynomial time algorithm that finds a 2 -approximation solution for MLSC $k$ problem.

Theorem 9 There exists a polynomial time algorithm that finds a 2-approximation solution for $M L S C k$.

\section{Conclusion}

In this paper we proved that the problem of covering a simple orthogonal art gallery with the minimum-length sliding $k$-transmitters, is NP-complete, even for $k=2$. 
Then, we presented a 2-approximation algorithm for this problem. The hardness of guarding an orthogonal polygon with the minimum cardinality sliding cameras and covering a polygon with the minimum cardinality sliding $k$-transmitters remain open.

\section{Acknowledgment}

We would like to thank Masood Seddighin for his valuable and helpful suggestions.

\section{References}

[1] O. Aichholzer, R. Fabila-Monroy, D. Flores-Pealoza, T. Hackl, C. Huemer, J. Urrutia, and B. Vogtenhuber. Modem illumination of monotone polygons. In Proceedings of the 25th European Conference on Computational Geometry, 167-170, 2009.

[2] B. Ballinger, N. Benbernou, P. Bose, M. Damian, E. D. Demaine, V. Dujmovic, R. Flatland, F. Hurtado, J. Iacono, A. Lubiw, P. Morin, V. Sacristan, D. Souvaine, R. Uehara. Coverage with $k$-transmitters in the presence of obstacles. Journal of Combinatorial Optimization, 25(2), 208-233, 2013.

[3] D. Beauquier, M. Nivat, E. Rmila, and J.M. Robson. Tiling pictures of the plane with two bars. Computational Geometry, 5(1): 1-25, 1995.

[4] S. Durocher, O. Filtser, R. Fraser, A. D. Mehrabi and S. Mehrabi. A (7/2)-Approximation Algorithm for Guarding Orthogonal Art Galleries with Sliding Cameras. In Proceedings of the 11th Latin American Symposium, 294-305, 2014.

[5] S. Durocher and S. Mehrabi. Guarding orthogonal art galleries using sliding cameras: algorithmic and hardness results. In Proceedings of the MFCS, LNCS 8087, 314-324, 2013.

[6] R. Fabila-Monroy, A. R. Vargas, and J. Urrutia. On modem illumination problems. In XIII Encuentros de Geometria Computacional, 2009.

[7] F. Hoffmann. On the rectilinear art gallery problem. In 17th International Colloquium on Automata, Languages and Programming, (ICALP), 717-728, 1990.

[8] M. J. Katz and G. Morgenstern. Guarding orthogonal art galleries with sliding cameras. International Journal of Computational Geometry and Applications, 21(2): 241-250, 2011.

[9] D. T. Lee and Arthur K. Lin. Computational complexity of art gallery problems. IEEE Transactions on Information Theory, 32(2): 276-282, 1986.

[10] R. Motwani, A. Raghunathan, and H. Saran. Covering orthogonal polygons with star polygons: The perfect graph approach. In Proceedings of the 4 th Symposium Computational Geometry, 211-223, 1988.

[11] J. O'Rourke. Art gallery theorems and algorithms. $O x$ ford University Press, Inc., New York, NY, USA, 1987.
[12] D. Schuchardt and H. D. Hecker. Two NP-hard artgallery problems for orthogonal polygons. Mathematical Logic Quarterly, 41(2): 261-267, 1995.

[13] J. Urrutia. Art gallery and illumination problems. In J.-R. Sack and J. Urrutia, editors, Handbook of Computational Geometry, 973-1027. North-Holland, 2000.

[14] C.Worman and J. M. Keil. Polygon decomposition and the orthogonal art gallery problem. International Journal of Computational Geometry and Applications., 17(2): 105-138, 2007. 